\title{
ESTUDO DE TRANSFORMAÇÕES SIMULTANEAS E SEQUENCIAIS VIA CONE CAUSAL*
}

Gabriel Moreira Kopke Júnior ${ }^{1}$ André Luiz Moraes Alves ${ }^{2}$ Weslley Luiz da Silva Assis ${ }^{3}$ Paulo Rangel Rios ${ }^{4}$

\section{Resumo}

As transformações sequenciais acontecem em vários materiais. Como exemplo temos a formação da Perlita e Ferrita devido a decomposição da Austenita, onde primeiro têm-se a formação de grãos de Ferrita e depois a nucleação dos grãos de Perlita. Em 2011, Rios e Villa desenvolveram um método analítico matematicamente exato para as transformações simultâneas e sequenciais. Neste trabalho usou-se a metodologia do Cone Causal para o modelamento microestrutural de reações simultâneas e sequenciais. Vale salientar que o método do Cone Causal apresenta grande versatilidade e gera resultados expressivos, como já apresentado em diversos trabalhos. A simulação nos permite obter parâmetros microestruturais quantitativos como: Fração volumétrica transformada, área superficial por unidade de volume, velocidade de avanço das interfaces, caminho microestrutural e outros. A principal motivação para a simulação é a geração de microestruturas virtuais simuladas que as transformações simultâneas e sequenciais podem apresentar.

Palavras-chave: Recristalização; Simulação Computacional; Reações Sequenciais.

\section{STUDY OF SIMULTANEOUS AND SEQUENTIAL TRANSFORMATIONS WITH} CONE CAUSAL

\section{Abstract}

The sequential transformations may take place in many materials. For example, the formation of perlite and ferrite by the decomposition of austenite. The first step of the decomposition of austenite is the formation of ferrite grains and the second step is the nucleation of perlite grains. In 2011, Rios and Villa developed a new analytical mathematical exact model for simultaneous and sequential transformations. The microstructural modeling of simultaneous and sequential transformations was treated in this work using the Causal Cone methodology. The Causal Cone method shows a big adaptability and create expressive results. Previous works already showed the efficiency of the Causal Cone methodology. By the computer simulation, one can obtain microstructural and qualitative parameters. For example: Transformed volume fraction, superficial area per unit volume, microestrutural path and others. The motivation in use computer simulation was that the chance to generate virtual simulated microstructures that the simultaneous and sequential transformations may present.

Keywords: Recrystallization; Computional Simulation; Sequential Reactions.

Eng. Metalúrgico, Graduando em Engenharia Metalúrgica na UFF, Volta Redonda, RJ, BR.

Eng. Metalúrgico, Doutorando em Engenharia Metalúrgica na UFF, Volta Redonda, RJ, BR.

Prof. Pós-Doutorado em Engenharia Metalúrgica, Prof. Adjunto do Curso de Engenharia Metalúrgica da UFF, Volta Redonda, RJ,BR

4 Prof. PhD em Engenharia Metalúrgica, Prof. Titular do curso de pós-graduação em Engenharia Metalúrgica da UFF, Volta Redonda, RJ,BR. 


\section{INTRODUÇÃO}

A cinética formal vem sendo empregada para o modelamento de transformações de fase em matéria condensada, mostrando ser eficaz na descrição de fenômenos do tipo. A metodologia da cinética formal começou com os trabalhos de Johnson[1], Mehl[2], Kolmogorov[3] e Avrami[4,5], Ela é também conhecida como modelo de JMAK.

As equações de JMAK são utilizadas para o modelamento da transformação de uma fase em outra ou no caso de recristalização, onde uma nova fase surge às custas da matriz anteriormente deformada. Porém, um outro caso que pode passar a existir é o surgimento de mais de um produto advindo de uma mesma fase matriz, sendo que estes produtos podem ser formados de maneira simultânea ou sequencial.

Reações simultâneas ocorrem quando ambos os produtos começam a se formar no mesmo tempo, ou seja, considerando a formação de duas fases produto, a nucleação de da fase 1 e fase 2 ocorrem no mesmo tempo, $t_{0}$. Poucos modelos são feitos com base em reações simultâneas.

Reações sequenciais ocorrem quando a nucleação da fase 1 e fase 2 ocorrem em tempos distintos. Sendo que a nucleação de uma fase 2 ocorrerá quando uma certa fração volumétrica da fase $1, V_{V 1}$, já tiver sido transformada.

Rios e Villa[6] desenvolveram um método analítico novo e matematicamente exato para modelar transformações simultâneas e sequenciais. Este novo método permite se construir modelos analíticos para duas ou mais reações a partir dos modelos individuais teóricos. Além disto, permite extrair a cinética das reações individuais a partir da cinética total de transformação. O método analítico fornece valores de parâmetros estereológicos, tais como: fração volumétrica, área por unidade de volume e caminho microestrutural.

Neste trabalho foi usado a metodologia do Cone Causal para a simulação de reações simultâneas e sequenciais gerando assim uma matriz com novos grãos de ambas as fases.

\section{MATERIAIS E MÉTODOS}

\subsection{Descrição das simulações}

Para este trabalho utilizou-se a metodologia do Cone Causal para simulação das reações simultâneas e sequenciais, sendo que estas reações ocorreram por nucleação e crescimento em ambas as fases. Nas simulações, parâmetros estereológicos foram alterados para termos alguns casos particulares.

Fez-se uso de um programa computacional escrito em linguagem Fortran 2003, e compilado pelo Microsoft Visual Studio 2012 §. Utilizou-se paralelização em Open MP. A visualização das microestruturas transformadas e de suas curvaturas foi realizada com o programa Tecplot $360^{\mathrm{TM}}$, os gráficos foram construídos com 0 auxílio do programa Wolfram Mathematica $10 \AA$.

Em todos os casos as transformações ocorreram em uma matriz cúbica que possuiu $300 \times 300 \times 300$, células cúbicas, onde as fases nuclearam e cresceram até ocuparem todo o espaço da matriz (100\% de transformação).

\subsection{Reações Sequenciais.}


Nesta secção demonstra-se as expressões analíticas para as reações sequenciais desenvolvidas por Rios e Villa[6], que foram comparadas com as simulações.

Supondo a existência de duas reações sequenciais chamadas de reação 1 e reação 2. A reação 1 ocorrerá no tempo, $t$, enquanto a reação 2 ocorrerá no tempo, $t_{2}$, sendo $t_{2}>t$. Os demais parâmetros da transformação foram considerados iguais em ambas as reações. Então as velocidades de crescimento são iguais, $G_{1}=G_{2}$. A nucleação em ambos os casos é feita por saturação de sítios, e os núcleos estão distribuídos aleatoriamente no espaço. O número de núcleos por unidade de volume também é igual para ambas as reações, $N_{V 1}=N_{V 2}$. A fração volumétrica de ambas as fases é descrita por:

Para $t>t_{2}$

$$
V_{V 1}(t)=1-\exp \left(-\frac{4 \pi}{3} N_{V 1} G_{1}^{3} t_{1}^{3}\right)
$$

$$
V_{V 2}(t)=1-\exp \left(-\frac{4 \pi}{3} N_{V 2} G_{2}^{3}\left(t-t_{2}\right)^{3}\right)
$$

Os valores experimentais para fração volumétrica, $V_{V}^{\text {exp }}$, para cada fase são calculados de acordo com as equações:

Para $t<t_{2}$

$$
\begin{aligned}
& V_{V 1}^{\text {exp }}=\int_{0}^{t} 4 \pi N_{V 1} G_{1}^{3} s^{2} \exp \left(-\frac{4 \pi}{3} N_{V 1} G_{1}^{3} s^{3}\right) d s \\
& V_{V 1}^{\text {exp }}=0
\end{aligned}
$$

Para $t>t_{2}$

$$
\begin{gathered}
V_{V 12}^{e x p}(t)=\int_{t}^{t_{2}} 4 \pi N_{V 1} G_{1}^{3} s^{2} \exp \left(-\frac{4 \pi}{3}\left(N_{V 1} G_{1}^{3} s^{3}+N_{V 2} G_{2}^{3}\left(s-s_{2}\right)^{3}\right)\right) d s \\
V_{V 22}^{\text {exp }}(t)=\int_{t}^{t_{2}} 4 \pi N_{V 2} G_{2}^{3}\left(s-s_{2}\right)^{2} \exp \left(-\frac{4 \pi}{3}\left(N_{V 1} G_{1}^{3} s^{3}+N_{V 2} G_{2}^{3}\left(s-s_{2}\right)^{3}\right)\right) d s
\end{gathered}
$$

A fração volumétrica total pode ser obtida por:

Para $t<t_{2}$ :

$$
V_{V}^{\text {exp }}(t)=V_{V 1}^{\text {exp }}(t)+V_{V 2}^{\text {exp }}(t)
$$

Para $t>t_{2}$ :

$$
V_{V}^{\text {exp }}(t)=V_{V 12}^{\text {exp }}(t)+V_{V 22}^{\text {exp }}(t)
$$

A quantidade de área interfacial por unidade de volume, $S_{V}^{\exp }$, entre o material transformado e o não transformado para cada fase pode ser expresso da seguinte forma:

Para $t<t_{2}$ :

$$
\begin{aligned}
& S_{V 1}^{e x p}(t)=4 \pi N_{V 1} G_{1}^{2} t^{2} \exp \left(-\frac{4 \pi}{3} N_{V 1} G_{1}^{3} t^{3}\right) \\
& S_{V 2}^{\text {exp }}(t)=0
\end{aligned}
$$

Para $t>t_{2}$ :

$$
\begin{array}{r}
S_{V 12}^{\exp }(t)=4 \pi N_{V 1} G_{1}^{2} t^{2} \exp \left(-\frac{4 \pi}{3}\left(N_{V 1} G_{1}^{3} t^{3}+N_{V 2} G_{2}^{3}\left(t-t_{2}\right)^{3}\right)\right) \\
S_{V 22}^{\exp }(t)=4 \pi N_{V 2} G_{2}^{2}\left(t-t_{2}\right)^{2} \exp \left(-\frac{4 \pi}{3}\left(N_{V 1} G_{1}^{3} t^{3}+N_{V 2} G_{2}^{3}\left(t-t_{2}\right)^{3}\right)\right)
\end{array}
$$

As quantidades totais obtidas serão:

$$
\begin{aligned}
& \text { Para } t<t_{2}: \\
& S_{V}^{\text {exp }}(t)=S_{V 1}^{\text {exp }}(t)+S_{V 2}^{\text {exp }}(t) \\
& \text { Para } t>t_{2}: \\
& S_{V}^{\text {exp }}(t)=S_{V 12}^{\text {exp }}(t)+S_{V 22}^{\text {exp }}(t)
\end{aligned}
$$


Podemos estimar a velocidade, $G$, por:

$$
G(t, x)=\frac{1}{s_{V}(t, x)} \frac{\partial V_{V}(t, x)}{\partial t}
$$

O caminho microestrutural pode ser estimado realizando-se uma plotagem paramétrica entre as equações de $S_{V}$ e $V_{V}$, para essa operação vale ressaltar que, as equações de $S_{v}$ e $V_{v}$ de cada fase mudam com o tempo, $t$.

Neste caso optou-se por não mostrar as equações simultâneas pois são consideradas um caso particular de transformações sequenciais, onde as fases nucleiam no mesmo tempo e possuem a mesma velocidade de crescimento. Isso é mostrado em [6].

\section{RESULTADOS E DISCUSSÃO}

Como resultados foram construídos gráficos com os dados da simulação, que são representados por pontos, e curvas geradas pelas equações analíticas citadas anteriormente, que são plotadas como curvas contínuas. Para melhorar a leitura e interpretação dos gráficos nem todos os pontos da simulação foram plotados.

Todas as simulações foram feitas por saturações de sítios com núcleos distribuídos aleatoriamente no espaço tanto para fase 1 quanto para fase 2. A nucleação da fase 2 ocorre quando a fase 1 alcança $V_{V}=0.1$, e ambas as fases possuem a mesma velocidade de crescimento.

A figura 1 mostra a microestrutura simulada de uma transformação envolvendo duas fases produtos de maneira simultânea. A fase 1 em azul e fase 2 em verde. É fácil notar que ambas as fases nucleiam no mesmo tempo.

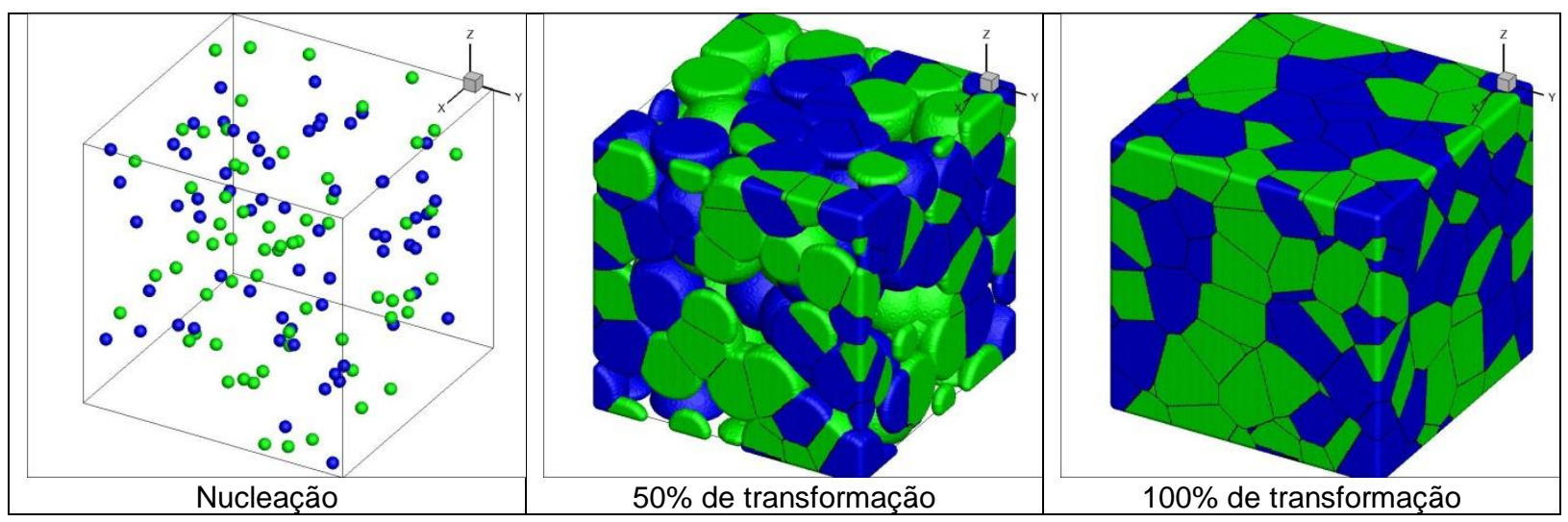

Figura 1. Simulação de uma microestrutura gerada por reações simultâneas.

A figura 2 mostra a microestrutura simulada de uma transformação envolvendo duas fases produtos de maneira sequencial. Novamente tem-se a fase 1 em azul e fase 2 em verde. Nota-se que a nucleação ocorre em tempos distintos entre as fases, e como dito anteriormente a fase 2 só surgiu quando a $V_{V}=0.1$. 


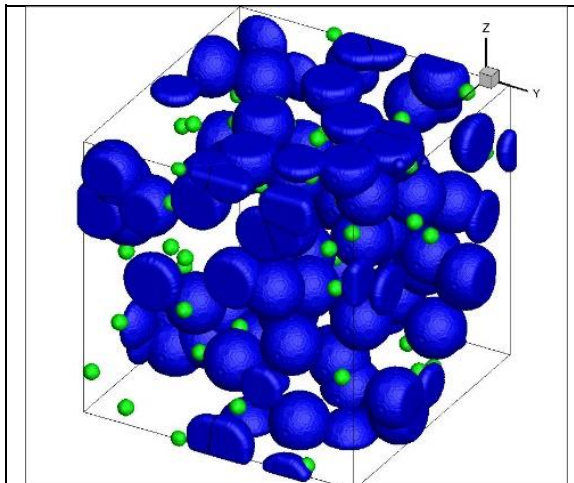

Nucleação da fase 2, com $V_{v}=0.1$

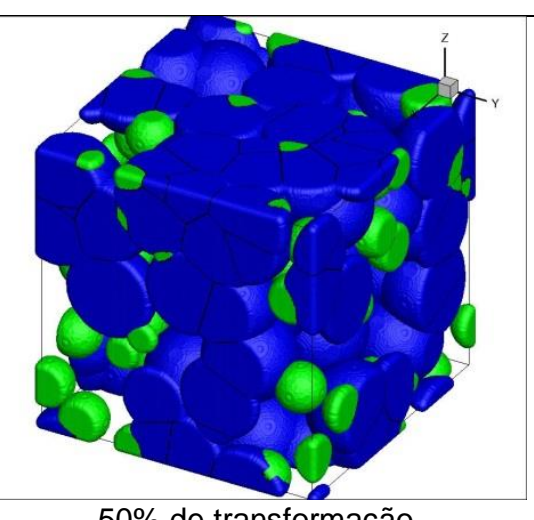

$50 \%$ de transformação

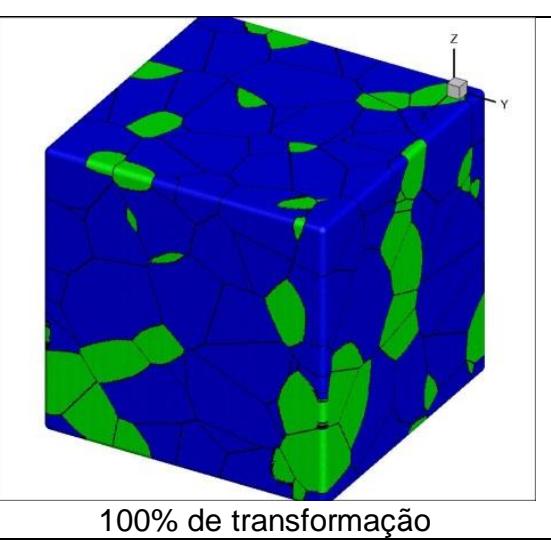

$100 \%$ de transformação

Figura 2. Simulação de uma microestrutura gerada por reações sequenciais

$\mathrm{Na}$ figura 3, mostra-se o plano superior da microestrutura formada em cada transformação, sendo possível observar os contornos entre os grãos de mesma fase e grãos de fases distintas.

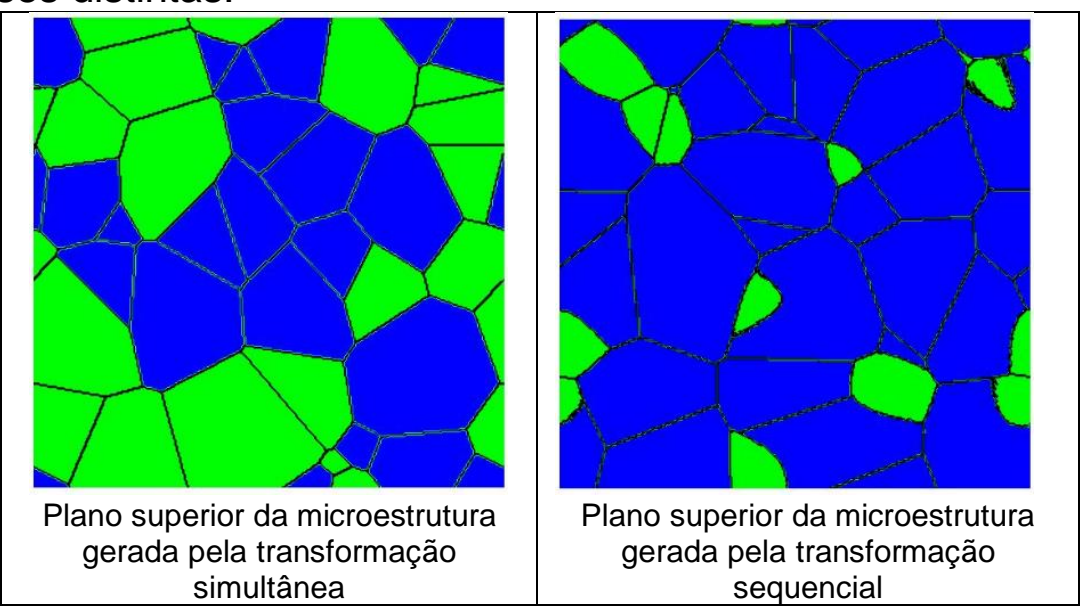

Figura 3. Planos superiores das microestruturas obtidas por reações simultâneas e sequenciais

As figuras 4 e 5 mostram a evolução da fração volumétrica transformada, $V_{V}$, com o tempo para as transformações simultâneas e sequenciais, respectivamente. Novamente tem-se a fase $1 \mathrm{em}$ azul e fase 2 em verde, porém agora demonstra-se os resultados da transformação total, em preto. 


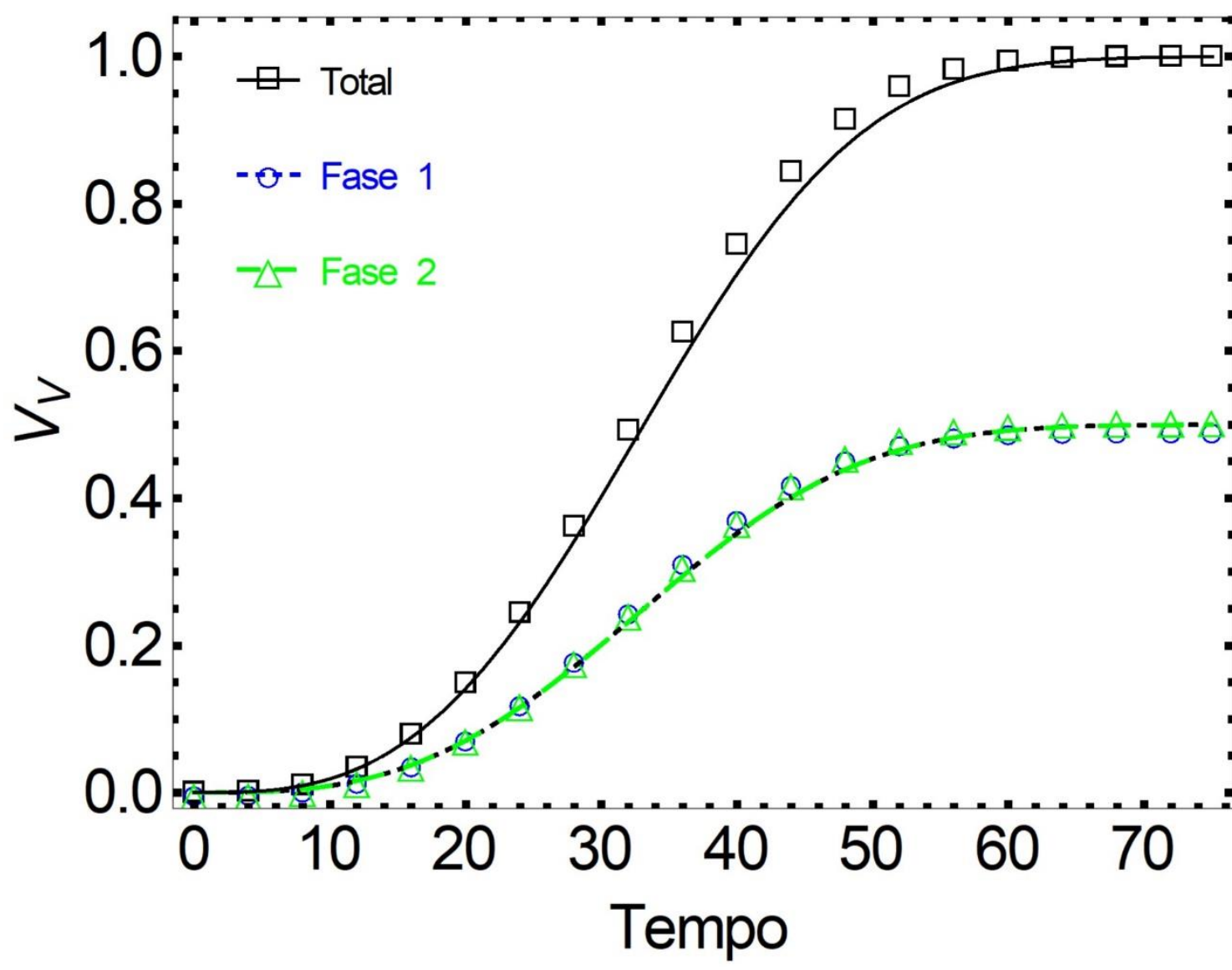

Figura 4: $V_{V}$ versus tempo para transformação simultânea com $G_{1}=G_{2}$ e $N_{1}=N_{2}=64$

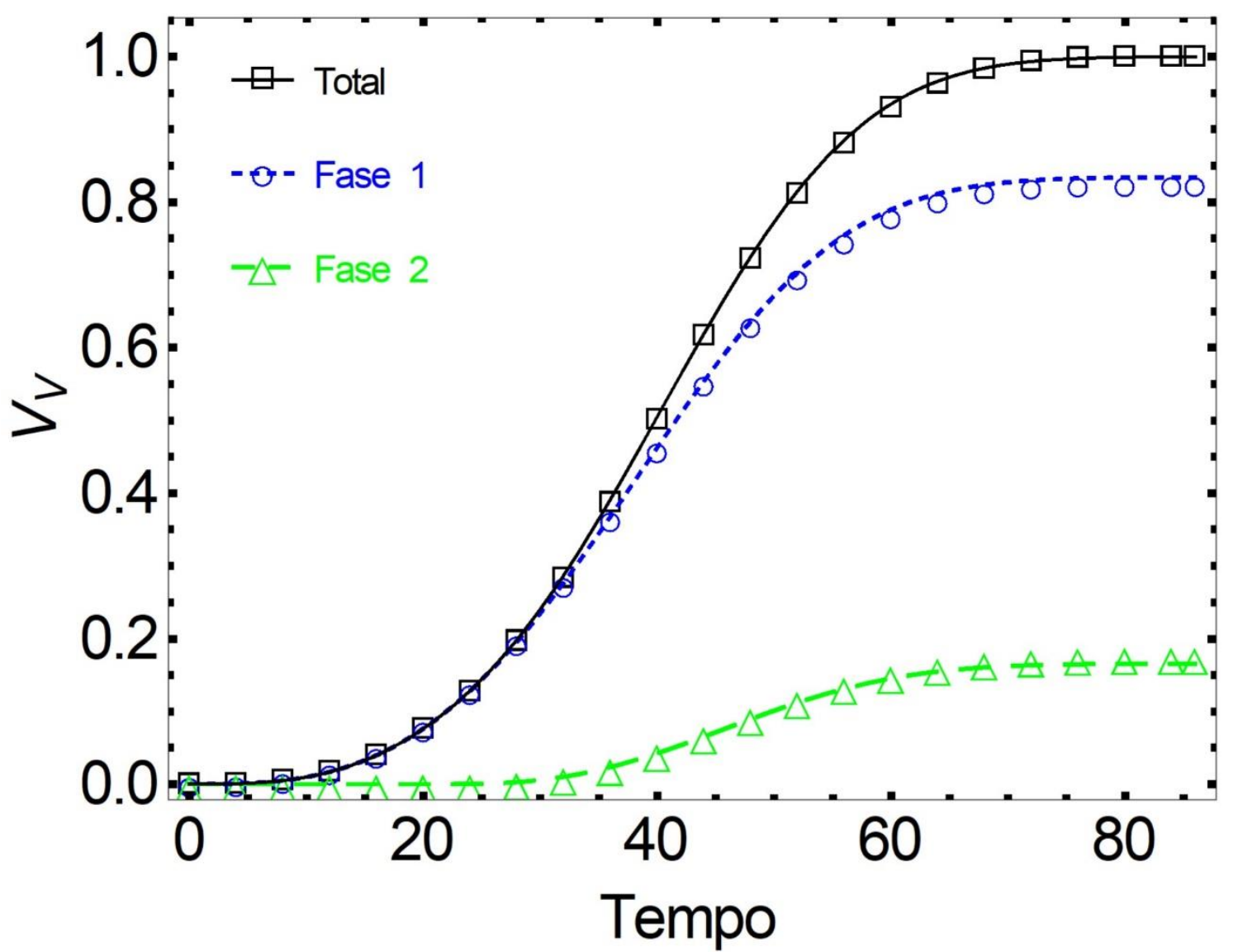

Figura 5: $V_{V}$ versus tempo para transformação sequencial com $G_{1}=G_{2}$ e $N_{1}=N_{2}=64$ 
Observa-se nas figuras 4 e 5, que na transformação sequencial a fase 2 apresenta menor influência, mostrando o domínio da fase 1. Esse domínio da fase 1 ocorre devido ao surgimento posterior da fase 2. Este resultado está de acordo com o modelo analítico de Rios e Villa[6].

As figuras 6 e 7 mostram a área interfacial entre material transformado e não transformado por unidade de volume contra a fração volumétrica transformada, $S_{V}$ versus $V_{V}$. As curvas descrevem o Caminho Microestrutural[10] de cada transformação.

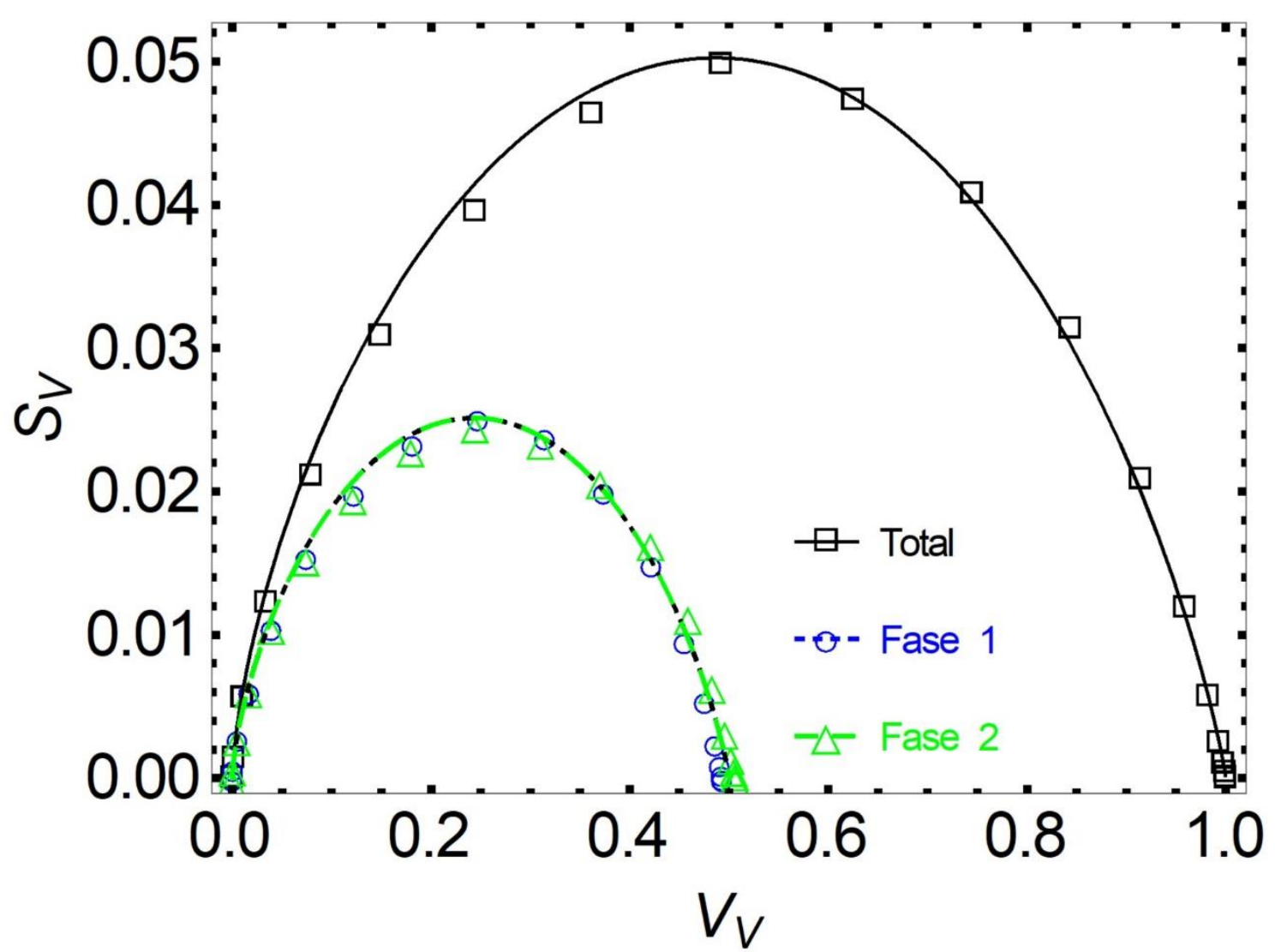

Figura 6: Caminho Microestrutural para transformação simultânea com $G_{1}=G_{2}$ e $N_{1}=N_{2}=64$ 


\section{Agradecimentos}

Agradecemos ao Conselho Nacional de Desenvolvimento Científico e Tecnológico, CNPq, a Coordenação de Aperfeiçoamento de Pessoal de Nível Superior, CAPES, e a Fundação de Amparo à Pesquisa do Estado do Rio de Janeiro, FAPERJ, pelo apoio financeiro.

\section{REFERÊNCIAS}

1 PRICE, C. W. Use of Kolmogorov-Johnson-Mehl-Avrami Kinetics in Recrystallization of Metals and Crystallization of Metallic Glasses Acta Metallurgica and Materialia. 1990, vol. 38, $n^{\circ} .5$, p. 727-738.

2 JOHNSON, W. A. AND MEHL, R. F, Metallurgical Transactions Soc. A.I.M.E. 1939, vol. 135, p.416-441.

3 KOLMOGOROV, A. N. Izv. Akad. Nauk. USSR-Ser-Matemat. 1937, vol. 1 (3), p.355.

4 AVRAMI, M. Journal of Chemical Physics. 1939, vol. 7, p.1103-1112.

$5 \quad$ AVRAMI, M. Journal of Chemical Physics. 1940, vol. 8, p. 212.

$6 \quad$ P. R. Rios, E. Villa: Acta Materialia. Vol. 59, 2011, p.1632-1643.

7 H.J. Frost, C.V. Thompson, The effect of nucleation conditions on the topology and geometry of two-dimensional grain structures, Acta Metall. 35 (1987) 529e540, http://dx.doi.org/10.1016/0001-6160(87)90258-6.

8 H.J. Frost, C.V. Thompson, Computer simulation of microstructural evolution in thin films, J. Electron. Mater 17 (1988) 447e458.

9 H.J. Frost, Microstructural evolution in thin films, Mater. Charact. 32 (1994) 257e273, http://dx.doi.org/10.1016/1044-5803(94)90102-3.

10 ALVES,ANDRÉ L.M. ; ASSIS, W.L.S. ;RIOS. . Computer simulation of sequential transformations. Acta Materialia (Oxford) v.126, p.451468,2017,http://dx.doi.org/10.1016/j.actamat.2016.12.068, 